\title{
Combined Therapeutic Effect of Probucol and Cilostazol on Endothelial Function in Patients with Silent Cerebral Lacunar Infarcts and Hypercholesterolemia: A Preliminary Study
}

\author{
Bonpei Takase $^{a}$ Masayoshi Nagata $^{c}$ Hidemi Hattori ${ }^{b}$ Yoshihiro Tanaka $^{b}$ \\ Masayuki Ishiharab \\ ${ }^{\mathrm{a}}$ Department of Intensive Care Medicine, and ${ }^{\mathrm{b}}$ Division of Biomedical Engineering, National Defense Medical \\ College Research Institute, National Defense Medical College, Tokorozawa, and 'Iruma Heart Hospital, Iruma, Japan
}

\section{Key Words}

Flow-mediated vasodilatation - Dyslipidemia .

Atherosclerosis · Antiplatelet agents

\begin{abstract}
Objective: This study evaluated the efficacy of combined therapy with probucol and cilostazol on endothelial function in silent lacunar cerebral infarcts (SLCl) and mild hypercholesterolemia. Subjects and Methods: Flow-mediated vasodilatation (FMD) and nitroglycerin-induced vasodilatation (NMD) were measured before and after 4 weeks of combined therapy with probucol ( $500 \mathrm{mg} /$ day) and cilostazol $(200 \mathrm{mg} /$ day) in 34 patients with a mean age of $72 \pm 7$ years (range 57-80 years) with SLCl, mild hypercholesterolemia (low-density lipoprotein cholesterol $>100 \mathrm{mg} / \mathrm{dl}$ ) and impaired endothelial function (FMD $<6 \%$ ). Patients were randomly allocated to one of the following two treatment groups: (1) aspirin (100 mg/day) with behavioral modifications, such as diet and/or exercise therapy (A group or control group, $n=17$ ), and (2) probucol and cilostazol treatment (PC group, $n=17$ ), also with behavioral modifications. Results: Although the baseline FMD was not different between the two treatment arms ( $2.7 \pm 1.5$ vs. $2.6 \pm 1.5 \%$, n.s.), the posttreatment FMD
\end{abstract}

was significantly improved in the PC group (from $2.7 \pm 1.5$ to $3.5 \pm 1.7 \%, \mathrm{p}<0.05$ ) but not in the A group (from $2.6 \pm 1.5$ to $2.9 \pm 1.4 \%$, n.s.). No differences were observed between baseline and posttreatment NMD in either group. The effects of treatments on lipid profiles were more profound in the PC group. Conclusion: Combined treatment with probucol and cilostazol resulted in subacute improvement in FMD/endothelial function in patients with SLCl with mild hypercholesterolemia. This combination therapy has the potential to reduce the risk of cardiovascular events via improvements in endothelial function and lipid profiles.

๑) 2013 S. Karger AG, Basel

\section{Introduction}

Therapies that lower low-density lipoprotein cholesterol (LDL-C) are critical for the secondary prevention of acute coronary syndrome or other cardiovascular events, including stroke [1]. Silent lacunar cerebral infarcts (SLCI) complicated by hypercholesterolemia are associated with poor cardiovascular outcomes [2]. Among the therapies that lower LDL-C, there is strong evidence that statins are useful for the secondary prevention of acute

Bonpei Takase, MD

3-2 Namiki

Tokorozawa, Saitama 359-8513 (Japan)

E-Mail bonpeit@ndmc.ac.jp 
coronary syndrome or ischemic cerebral infarction. However, statin therapy cannot be applied in all patients due to its side effects, such as liver dysfunction and myositis [1].

Probucol is a mild cholesterol-lowering agent that has antioxidant, anti-inflammatory and antiatherosclerotic properties [3]. Since probucol causes a significant reduction in high-density lipoprotein cholesterol (HDL-C) and prolongation of the QT interval, it has been withdrawn from the market in some countries [3]. However, studies suggest that probucol reduces atherosclerosis and prevents restenosis after percutaneous coronary intervention [3] and that it may produce a synergistic antiatherosclerotic effect when administered with cilostazol [4]. Cilostazol is an inhibitor of the type 3 phosphodiesterase that has antiplatelet and diverse antiatherogenic properties. Previous reports have shown that cilostazol improves endothelial function and attenuates antioxidant stress through an increase in nitric oxide production [5] and scavenging of free radicals [6], respectively. Cilostazol also inhibits foam cell formation [7] and smooth muscle cell proliferation [8].

Endothelial dysfunction is present in both the early and the advanced phase of atherosclerosis or the destabilized phase of atherosclerotic disorders [9]. Since endothelial function plays a central role in the pathogenesis of atherosclerotic progression, the synergistic effect of probucol and cilostazol on endothelial function is worth being evaluated in patients who are at high risk for atherosclerotic events, such as those with SLCI.

Endothelial function can be investigated through the assessment of brachial artery flow-mediated vasodilatation (FMD) or plethysmography with acetylcholine infusion in the forearm vessels. The FMD of the brachial artery combined with nitroglycerin-mediated vasodilatation (NMD) is frequently used to evaluate endothelial function [10].

Thus, the purpose of the present study was to investigate the effect of combined therapy with probucol and cilostazol on endothelial function in patients with SLCI and hypercholesterolemia.

\section{Subjects and Methods}

\section{Study Population}

The study population consisted of 34 male patients (mean age $72 \pm 7$ years; range $57-80$ years) with hypercholesterolemia and SLCI who were referred to our hospital. Patients were included in this study if their LDL-C was $>100 \mathrm{mg} / \mathrm{dl}$ despite having already been prescribed lipid-lowering medication, including statins or other lipid-lowering agents. The SLCI was diagnosed by magnetic resonance imaging (MRI) with a Signa 1.5-T Cvi scanner (GE Medical Systems, Milwaukee, Wisc., USA) and a commercially available phased-array brain coil. Brain transverse proton densityweighted and T1-weighted and T2-weighted images were obtained, and SLCI was defined as focal T2 hyperintensities $>3 \mathrm{~mm}$ with correlative T1 hypointensities [11].

Twenty-one of the study patients had coronary artery disease, and 17 were receiving statin therapy before entering this study. In these patients, statins were withdrawn for at least 5 half-lives of the respective statin before the initiation of probucol. In addition, aspirin had been prescribed to 24 of the patients. In these patients, aspirin was discontinued for at least 7 days before entry into this study. Exclusion criteria were: allergic reaction to probucol, cilostazol or aspirin; diabetic acidosis; advanced heart failure and arrhythmias; thyroid disease; severe disease of the liver and kidney; pregnancy, or any other acute disorder. In this study, the comorbid conditions were defined as follows: hypertension (either systolic blood pressure $>160 \mathrm{~mm} \mathrm{Hg}$ or diastolic blood pressure $>90 \mathrm{~mm}$ $\mathrm{Hg}$ ); coronary artery disease (defined as in the text); diabetes mellitus (fasting blood sugar $>126 \mathrm{mg} / \mathrm{dl}$ ), and peripheral artery disease (ankle-brachial index $<0.9$ following the confirmation of luminal stenosis of the peripheral artery by conventional imaging modality). Written informed consent was obtained from each patient. The study protocol was approved by our Institutional Review Board.

\section{Study Protocol}

This was a prospective, open-label, single-blind, active control, randomized study, and FMD and NMD were measured before and after the treatments. Each eligible patient was randomly assigned to one of two 4-week treatment arms. Treatment was either combined probucol (500 mg/day) and cilostazol (200 mg/day) with additional behavioral modifications, such as diet and/or exercise therapy ( $\mathrm{PC}$ group, $\mathrm{n}=17$, mean age $72 \pm 15$ years, range $64-80$ years), or aspirin (100 mg/day) with the same behavioral modifications as those used in the PC group (A group or control group, $\mathrm{n}=17$, mean age $72 \pm 8$ years, range $57-78$ years). After overnight fasting, venous blood sampling (for measurement of blood chemistries) and brachial artery endothelial function testing were done. Blood sample testing included routine blood chemistries with liver and renal function tests, lipid profile, fasting blood sugar and complete blood counts according to standard methods. All measurements were conducted before and after each treatment.

\section{Ultrasound FMD and NMD Measurements in the Brachial}

Artery

All ultrasound studies were done in a temperature-controlled room $\left(25^{\circ} \mathrm{C}\right)$ with the subject in a fasted, resting and supine state from approximately 14:00 h to 17:00 h. All studies were performed by the same technician, who was blinded to any other clinical information, including the study protocol. Heavy meals, including a high-fat diet and caffeine-containing beverages, were prohibited beginning on the night before the study. Patients were not allowed to have lunch on the day of the ultrasound study. Blood pressure and heart rate were recorded from the left arm every 3 min with an automatic sphygmomanometer (BP-203; Nihon Korin, Tokyo, Japan) during the ultrasound procedure. Vasodilatation responses of the brachial artery were determined via the ultrasound technique using a semiautomatic device (EF18G; UNEX, Nagoya, Ja- 
pan). Briefly, the diameter of the brachial artery was measured from $\mathrm{B}$-mode ultrasound images using a $10-\mathrm{MHz}$ linear array transducer. Then, a blood pressure cuff was inflated to $50 \mathrm{~mm} \mathrm{Hg}$ above the systolic blood pressure over the proximal portion of the right forearm for $5 \mathrm{~min}$. The diastolic diameter of the brachial artery was determined semiautomatically using an instrument equipped with software for monitoring the brachial artery diameter. The changes in diastolic diameter were continuously recorded. Then, FMD was determined as the maximum change in diameter after cuff release normalized to the baseline diameter (\% of baseline diameter). After a 15-min interval to obviate any effect of reactive hyperemia, NMD was assessed. Baseline measurements of brachial artery diameter and flow velocity were again obtained, and $0.3 \mathrm{mg}$ of sublingual nitroglycerin was then administered. Three minutes later, the brachial artery diameter was recorded. NMD was defined as the percent change of the brachial artery diameter relative to the baseline diameter. These measurements were obtained using the EF18G device. Calculation of these values by the EF18G device in our laboratory showed that the intra- and interobserver variabilities (coefficient of variation) for repeated measures of diameter before and after reactive hyperemia in the brachial artery were $<3 \%[10,12]$.

\section{Statistical Analysis}

Data are expressed as means \pm SD. Student's t test was used to compare data before and after each treatment. Pearson's productmoment correlation was performed between the changes in FMD and those in LDL-C in response to each treatment. Differences or statistical values were considered significant at $\mathrm{p}<0.05$. Analyses were conducted using SPSS version 11 (SPSS Inc., Chicago, Ill., USA).

\section{Results}

\section{Patient Profile}

The clinical characteristics of the study population are given in table 1 . The patients had a relatively high risk for atherosclerosis in addition to hypercholesterolemia and SLCI. Twenty-one $(>60 \%)$ patients had coronary artery disease, $11(30 \%)$ patients were hypertensive, and 17 (50\%) patients were diabetic. Five (15\%) patients had peripheral artery disease. Clinical characteristics were comparable between the two groups. Age, prevalence of comorbidities and combination treatments were similar in the two groups. The mean patient age in each group was 72 years (elderly population). Seventeen (50\%) patients had already been prescribed statins. However, their LDLC levels were $\geq 100 \mathrm{mg} / \mathrm{dl}$, which met the inclusion criteria, so these patients were enrolled into this study. The average FMD was $2.6 \%$ in both groups, and the average NMD was $8 \%$ in both groups. None of the patients had FMD $\geq 6 \%$, which is the normal cutoff value in our laboratory (table 2; fig. 1).

Probucol, Cilostazol and Endothelial Function in SLCI
Table 1. Clinical characteristics of the study population

\begin{tabular}{lcc}
\hline & $\begin{array}{c}\text { PC group } \\
(\mathrm{n}=17)\end{array}$ & $\begin{array}{c}\text { A group } \\
(\mathrm{n}=17)\end{array}$ \\
\hline Age, years & $72 \pm 7$ & $72 \pm 8$ \\
Male/female ratio & $17 / 0$ & $17 / 0$ \\
Complications, $\mathrm{n}(\%)$ & & \\
$\quad$ Hypertension & $6(35)$ & $5(29)$ \\
$\quad$ Coronary artery disease & $11(65)$ & $10(59)$ \\
Diabetes mellitus & $8(47)$ & $9(53)$ \\
$\quad$ Peripheral artery disease & $3(18)$ & $2(12)$ \\
Combination treatment, n $(\%)$ & & \\
$\quad$ ACE-I or ARB & $14(82)$ & $13(76)$ \\
$\beta$-Blocker & $11(65)$ & $11(65)$ \\
Calcium channel blockers & $10(59)$ & $10(59)$ \\
Nitrates & $5(29)$ & $3(18)$ \\
Antiplatelet agents & $14(82)$ & $13(76)$ \\
Oral antidiabetic agents & $8(47)$ & $9(47)$ \\
\hline
\end{tabular}

ACE-I = Angiotensin-converting enzyme inhibitor; $\mathrm{ARB}=$ angiotensin II receptor blocker; antiplatelet agents = antiplatelet agents other than cilostazol.

\section{Changes in FMD and NMD}

The effects of each treatment on hemodynamics, FMD and NMD are summarized in table 2 . In contrast to the A group, the PC group showed a significantly increased heart rate after treatment. The PC group experienced a significant increase in FMD after treatment (table 2; fig. 1, 2) whereas the A group did not, despite no significant changes in baseline brachial artery diameter and NMD in either group. Seventeen (88\%) patients in the PC group, and only $6(35 \%)$ patients in the A group, experienced an increase in FMD after treatment.

\section{Changes in Blood Chemistries}

With the notable exception of lipid profiles, no changes were detectable in most parameters of blood chemistries in either treatment group (table 3). Combined treatment with probucol and cilostazol (PC group) resulted in a significant decrease in LDL-C of $31 \%$ (from 134 to 92 $\mathrm{mg} / \mathrm{dl}$ ). Further, serum levels of total cholesterol decreased and HDL-C significantly decreased in the PC group. Changes in triglycerides were not statistically significant (table 3).

No significant relationship was found between the effect of combined treatment with probucol and cilostazol on FMD and that on LDL-C (delta FMD by treatment in the PC group vs. delta LDH-C by treatment in the PC group, $r=-0.13, \mathrm{n}=17$, n.s.). 


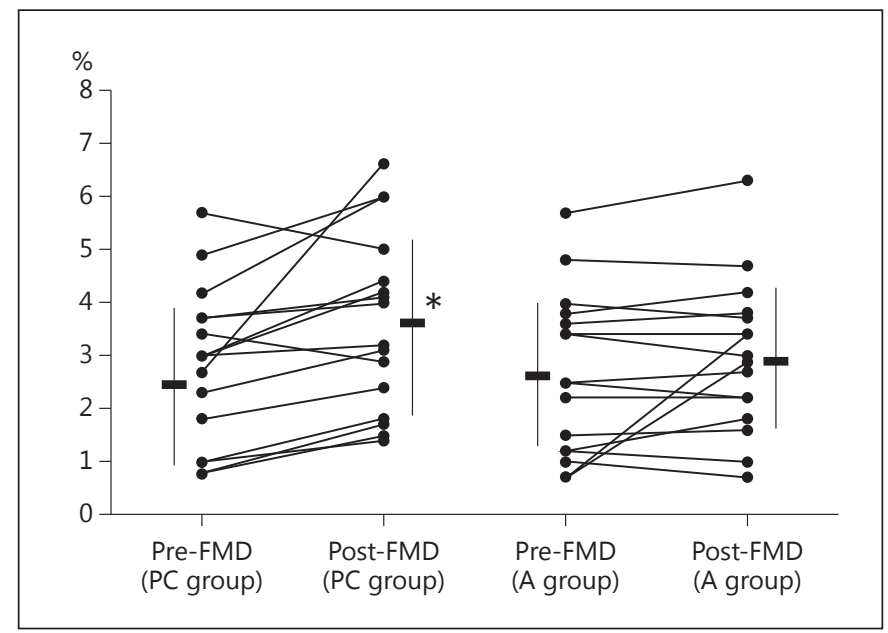

Fig. 1. Effect of each treatment on FMD. ${ }^{*} \mathrm{p}<0.05$ vs. Pre-FMD (PC group). Data are presented as means $\pm \mathrm{SD}$.

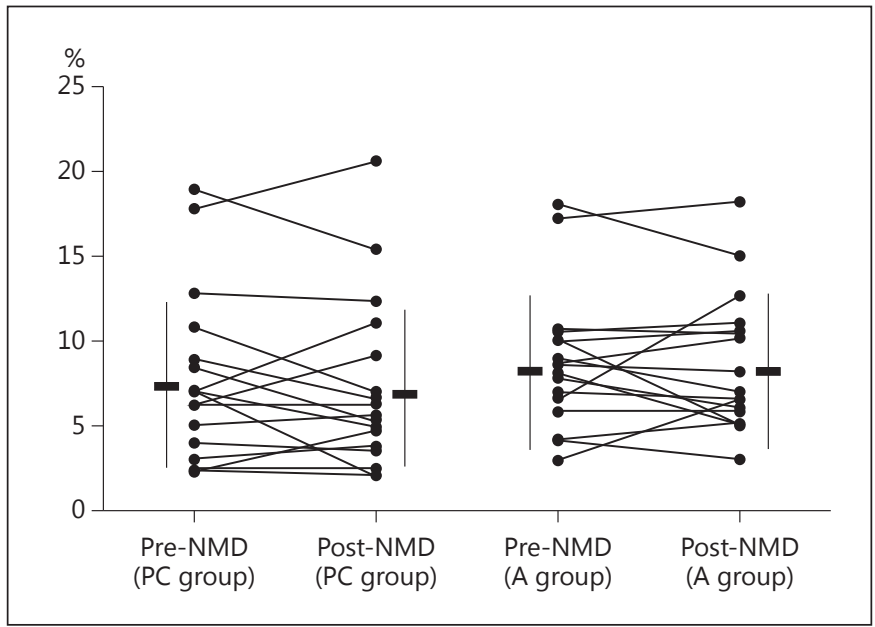

Fig. 2. Effect of each treatment on NMD. Data are presented as means \pm SD.

Table 2. Summary of ultrasound measurements for FMD and NMD in the brachial artery and the effect of each treatment

\begin{tabular}{|c|c|c|c|c|}
\hline & \multicolumn{2}{|c|}{ PC group $(n=17)$} & \multicolumn{2}{|c|}{ A group $(n=17)$} \\
\hline Diastolic BP, mm Hg & $71 \pm 9$ & $72 \pm 9$ & $72 \pm 10$ & $71 \pm 9$ \\
\hline Heart rate, beats/min & $60 \pm 11$ & $66 \pm 11^{*}$ & $61 \pm 10$ & $60 \pm 12$ \\
\hline Brachial artery diameter at baseline, $\mathrm{mm}$ & $4.67 \pm 0.56$ & $4.78 \pm 0.40$ & $4.52 \pm 0.57$ & $4.52 \pm 0.61$ \\
\hline
\end{tabular}

$\mathrm{BP}=$ Blood pressure. $^{*} \mathrm{p}<0.05$ vs. pretreatment.

Table 3. Effect of each treatment on lipid profiles and blood chemistry

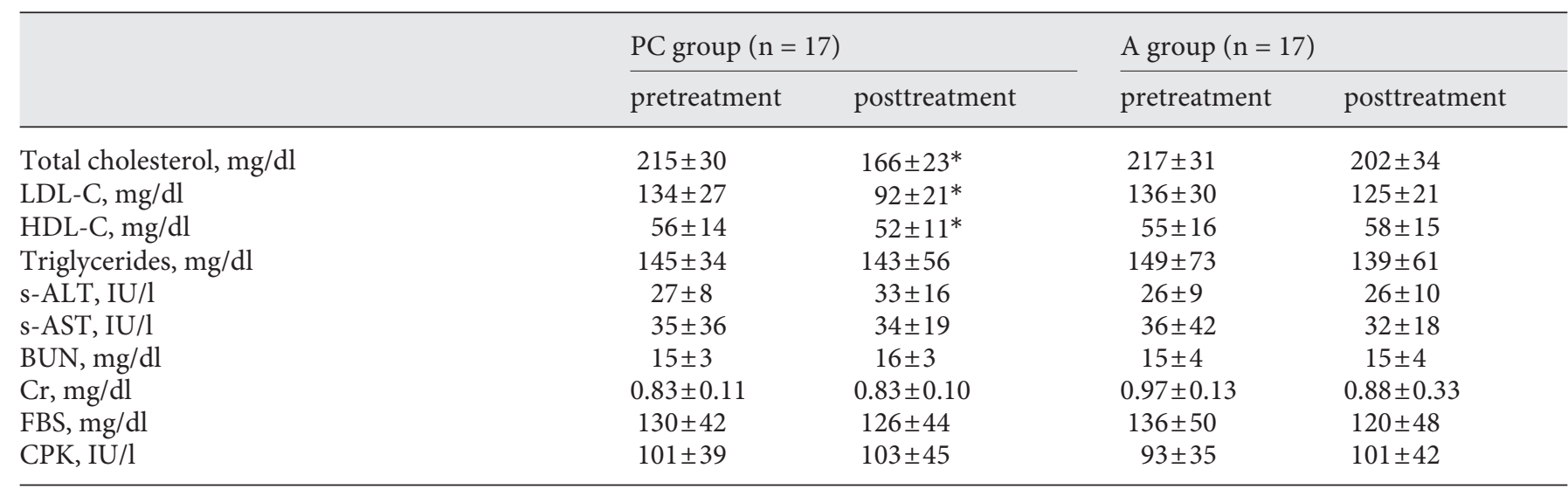

s-ALT = Serum alanine aminotransferase; s-AST = serum aspartate aminotransferase; BUN = blood urea nitrogen; Cr = serum creatinine; FBS $=$ fasting blood sugar; $\mathrm{CPK}=$ creatine phoshphokinase. ${ }^{*} \mathrm{p}<0.05$ vs. pretreatment. 


\section{Discussion}

The present study demonstrated that combined therapy with probucol and cilostazol resulted in subacute improvement in endothelial function and a decrease in LDL-C. Four weeks of treatment with probucol and cilostazol significantly improved the FMD without changing the NMD, suggesting that this therapy resulted in an improvement in brachial artery endothelial function. Changes in FMD in response to probucol and cilostazol did not correlate with changes in LDL-C. Thus, the effect of probucol and cilostazol on endothelial function is likely independent of that on LDL-C levels.

This is the first report to show a favorable effect of combined therapy with probucol and cilostazol on brachial artery FMD in patients with SLCI, hypercholesterolemia and other atherosclerotic risk factors (table 1). Since previous reports have shown that brachial artery FMD correlates with coronary endothelial function [10, 13 ] and that improvements in FMD play an important role in preventing the progression of atherosclerosis and the destabilization of atherosclerotic plaque $[9,14]$, the therapeutic effect of a 4 -week course of combined therapy with probucol and cilostazol is relevant to daily clinical practice. Endothelial dysfunction combined with plaque destabilization is a risk factor for cardiovascular events, such as acute coronary syndrome. The relatively quick action of combined therapy with probucol and cilostazol is another beneficial feature of this regimen.

Since the risk of developing overt stroke, dementia, depression and aspiration pneumonia is closely associated with SLCI [15], ameliorating the atherosclerotic burden in patients with multiple atherosclerotic risk factors is a clinically important goal. In this study population, more than $60 \%$ of patients suffered from coronary artery disease, $50 \%$ had diabetes and approximately $20 \%$ had peripheral artery disease. Since SLCI complicated with hypercholesterolemia has been reported to be associated with poor cardiovascular outcomes, and the impaired endothelial function reflected by decreased FMD values correlates with this increased risk, the salutary effect of probucol and cilostazol combined therapy on FMD can be considered to provide useful information to clinicians who treat SLCI patients with hypercholesterolemia. With the general aging of the population and the more widespread use of MRI, the prevalence of a diagnosis of SLCI is likely to increase.

Because of the prognostic role of SLCI [16] and the fact that endothelial function is a common pathway connecting atherosclerotic risk factors with the progression of atheromatous plaques and lesions, the effective treatment for endothelial function as shown in this study, that is combination therapy of probucol and cilostazol, is expected to improve the untoward outcome in patients with lacunar infarction. Lacunar infarction has been reported to be mainly caused by occlusion of a single penetrating artery, and pathophysiological studies revealed that this occlusion is brought about by either microatheromatosis or lipohyalinosis. In general, lacunar infarction shows a favorable prognosis in the short term, whereas it increases the risk of death, stroke recurrence and dementia in the mid and long term.

Since statin treatment has already been reported to improve endothelial function, combined therapy of probucol and cilostazol could be a potential alternative treatment to statins in patients with lacunar infarction. The role of statin treatment for either primary or secondary prevention of ischemic cardiovascular events including ischemic stroke has been widely accepted [17]. In addition, pretreatment with statins improves the early outcome in patients with first-ever ischemic stroke [18], and a recent meta-analysis on statin treatment and functional outcome after ischemic stroke [19] showed that statin therapy is associated with improved early and late outcomes in stroke [20,21].

The efficacy of probucol or cilostazol in endothelial function is consistent with that described in previous reports $[22,23]$. In many reports on experimental materials [30] and clinical populations [4], probucol or cilostazol improved endothelial function through decreases in oxidant stress [24] or upregulated endothelial nitric oxide synthase (eNOS) activity [25], respectively. In addition, previous reports have demonstrated that combined therapy with probucol and cilostazol reduced restenosis after percutaneous coronary intervention more effectively than either cilostazol or probucol alone. In addition, recent reports demonstrated the potential synergistic effects of cilostazol and probucol when they were used in combination for the prevention of atherosclerosis [27, 28]. In one recent study [27], the combined administration of cilostazol and probucol resulted in a greater decrease in the atherosclerotic lesion than when either drug was administered separately in LDL receptor-deficient mice.

It might be important to measure the plasma level of apolipoprotein-E when the effect of probucol on atherosclerotic lesions in patients with hyperlipidemia is studied, especially if type III hyperlipidemia is suspected in the study population. The reason apolipoprotein-E measurement is necessary is that probucol has been reported 
to have a strong proatherogenic effect in apolipoproteinE-deficient mice despite a reduction in the total plasma cholesterol levels [29], even though the results are still conflicting. Apolipoprotein combines with lipids to form lipoprotein particles. Thus, apolipoproteins are carrier proteins, and several classes of lipoproteins exist. Among these different lipoproteins, apolipoprotein-E is important when the antiatherosclerotic effect of probucol is studied, as shown above. Three different isoforms of apolipoprotein-E have been reported. These isoforms include apo-E2, apo-E3 and apo-E4. Especially apo-E2 is connected with type III hyperlipidemia where the blood levels of both LDL-C and triglyceride increase. In the present study, the plasma level of apo-E was not measured because none of the patients in this study showed the feature of type III hyperlipidemia, as shown in table 3. In this study population, LDL-C was abnormally high whereas the levels of triglycerides were not robustly high enough for type III hyperlipidemia to be diagnosed.

This study has several limitations. First, the number of patients was small, and all patients were men. In addition, the study was conducted in only two medical centers, including one academic medical school hospital. Thus, this study should be duplicated with a randomized, multicenter clinical trial design. Second, the mechanisms of the effect of the combined therapy of probucol and cilostazol were not investigated in the present study. However, as mentioned above, many previous experimental and clinical studies [26] have suggested possible mechanisms for the therapeutic benefit of these drugs. Third, the combined therapeutic effect of probucol and cilostazol on cognitive impairment was not evaluated in the present study. In addition, the effect of this therapy on the relationship between endothelial function and mild cognitive impairment was not evaluated. Since the pathological and morphological features on MRI subcortical hyperintensities have been reported to correlate with mild cognitive impairment of the vascular type in patients with lacunar infarction [30], the improvement of endothelial function with this therapy could possibly prevent the progression of subcortical hyperintensities and could subsequently preclude the cognitive aggravation in patients with lacunar infarction. Also, neuopsychological evaluations were not carried out in this study. These parameters should be investigated in future studies. Lastly, only the subacute effect of combined therapy with probucol and cilostazol was investigated. Therefore, the long-term effect of this regimen would benefit from further study. Indeed, the SECURE trials have already been started [4], and additional information regarding combined therapy with probucol and cilostazol should be available in the near future.

\section{Conclusion}

Combined therapy with probucol and cilostazol resulted in improved endothelial function in patients with SLCI, hypercholesterolemia and many other atherosclerotic risk factors. These results suggest that combined therapy with probucol and cilostazol might be a powerful antiatherosclerotic regimen and may improve long-term outcomes. Large-scale, randomized clinical trials are needed to confirm this conclusion.

\section{References}

1 Baigent C, Keech A, Kearney PM, et al, Cholesterol Treatment Trialists' (CTT) Collaborators: Efficacy and safety of cholesterol-lowering treatment: prospective meta-analysis of data from 90,056 participants in 14 randomized trials of statins. Lancet 2005;366:12671278.

-2 Bernick C, Kuller L, Dulberg C, et al, Cardiovascular Health Study Collaborative Reseach Group: Silent MRI infarcts and the risk of future stroke: the cardiovascular health study. Neurology 2001;57:1222-1229.

3 Yamashita S, Matsuzawa Y: Where are we with probucol: a new life for an old drug? Atherosclerosis 2009;207:16-23.
-4 Ko YG, Kim BK, Lee BK, et al, SECURE Investigators: Study design and rationale of 'Synergistic effect of combination therapy with cilostazol and ProbUcol on plaque stabilization and lesion REgression (SECURE)' study: a double-blind randomised controlled multicenter clinical trial. Trials 2011;12:10, $1-8$.

5 Nakamura T, Houchi H, Minami A, et al: Endothelium-dependent relaxation by cilostazol, a phosphodiesteras III inhibitor, on rat thoracic aorta. Life Sci 2001;69:17091715.

6 Kim KY, Shin HK, Choi JM, et al: Inhibition of lipopolysaccharide-induced apoptosis by cilostazol in human umbilical vein endothelial cells. J Pharmacol Exp Ther 2002;300: 709-715.
7 Okutsu R, Yoshikawa T, Nagasawa M, et al: Cilostazol inhibits modified low-density lipoprotein uptake and foam cell formation in mouse peritoneal macrophages. Atherosclerosis 2009;204:405-411.

-8 Ishizaka N, Taguchi J, Kimura Y, et al: Effects of a single local administration of cilostazol on neointimal formation in balloon-injured rat carotid artery. Atherosclerosis 1999;142: 41-46.

-9 Aukrust P, Halvorsen B, Yndestad A, et al: Chemokines and cardiovascular risk. Arterioscler Thromb Vasc Biol 2008;28:1909-1919.

10 Takase B, Uehata A, Akima T, et al: Endothelium-dependent flow-mediated vasodilation in coronary and brachial arteries in suspected coronary artery disease. Am J Cardiol 1998; 82:1535-1539. 
11 Vermeer SE, Longstreth WT Jr, Koudstaal PJ: Silent brain infarcts: a systematic review. Lancet Neurol 2007;6:611-619.

-12 Tomiyama H, Matsumoto C, Yamada J, et al: The relationships of cardiovascular disease risk factors to flow-mediated dilatation in Japanese subjects free of cardiovascular disease. Hypertens Res 2008;31:2019-2025.

$\checkmark 13$ Takase B, Hamabe A, Satomura K, et al: Beneficial effects of nifedipine on vasodilator response to acetylcholine in coronary and brachial arteries in the patients with coronary artery disease. Int J Cardiol 2006;113:305310.

14 Szmitko PE, Verma S: C-reactive protein and statins: IL-8 as a molecular link? Clin Sci (Lond) 2005;108:493-495.

15 Lim JS, Kwon HM: Risk of 'silent stroke' in patients older than 60 years: risk assessment and clinical perspectives. Clin Interv Aging 2010;5:239-251.

16 Arboix A, Martí-Vilalta JL: Lacunar stroke. Expert Rev Neurother 2009;9:179-196.

17 Jun M, Foote C, Lv J, et al: Effects of fibrates on cardiovascular outcomes: a systematic review and meta-analysis. Lancet 2010;375: 1875-1884.

18 Arboix A, García-Eroles L, Oliveres M, et al: Pretreatment with statins improves early outcome in patients with first-ever ischaemic stroke: a pleiotropic effect of statins or a beneficial effect of hypercholesterolemia? BMC Neurol 2010;10:47.
19 NíChróinín D, Callaly EL, Duggan J, et al: Association between acute statin therapy, survival, and improved functional outcome after ischemic stroke: the North Dublin Population Stroke Study. Stroke 2011;42:1021-1029.

$20 \mathrm{Kim} \mathrm{KH}$, Cho SH, Yim YR, et al: Effects of low dose versus high dose statin therapy on the changes of endothelial function and carotid intima-media thickness in patients with variant angina. J Cardiovasc Ultrasound 2013;21: 58-63.

21 Akanji AO: Diabetic dyslipidaemia in Kuwait. Med Princ Pract 2002;11(suppl 2):47-55.

22 Kim KS, Park HS, Jung IS, et al: Endothelial dysfunction in the smokers can be improved with oral cilostazol treatment. J Cardiovasc Ultrasound 2011;19:21-25.

23 Oida K, Ebata K, Kanehara H, et al: Effect of cilostazol on impaired vasodilatory response of the brachial artery to ischemia in smokers. J Atheroscler Thromb 2003;10:93-98.

24 Park SY, Lee JH, Shin HK, et al: Synergistic efficacy of concurrent treatment with cilostazol and probucol on the suppression of reactive oxygen species and inflammatory markers in cultured human coronary artery endothelial cells. Korean J Physiol Pharmacol 2008; $12: 165-170$.
25 Santos MR, Celotto AC, Capellini VK, et al: The protective effect of cilostazol on isolated rabbit femoral arteries under conditions of ischemia and reperfusion: the role of the nitric oxide pathway. Clinics (Sao Paulo) 2012;67: 171-178.

26 Park SY, Lee JH, Kim CD, et al: Beneficial synergistic effects of concurrent treatment with cilostazol and probucol against focal cerebral ischemic injury in rats. Brain Res 2007;1157: 112-120.

27 Yoshikawa T, Mitani K, Kotosai K, et al: Antiatherogenic effects of cilostazol and probucol alone, and in combination in low density lipoprotein receptor-deficient mice fed with a high fat diet. Horm Metab Res 2008;40:473478.

28 Park SY, Lee JH, Shin HK, et al: Synergistic efficacy of concurrent treatment with cilostazol and probucol on the suppression of reactive oxygen species and inflammatory markers in cultured human coronary artery endothelial cells. Korean J Physiol Pharmacol 2008;12:165-170.

29 Yoshikawa T, Shimano H, Chen Z, et al: Effects of probucol on atherosclerosis of apoEdeficient or LDL receptor-deficient mice. Horm Metab Res 2001;33:472-479.

-30 Grau-Olivares M, Bartrés-Faz D, Arboix A, et al: Mild cognitive impairment after lacunar infarction: voxel-based morphometry and neuropsychological assessment. Cerebrovasc Dis 2007;23:353-361. 